\title{
Análisis del impacto de la Ley Orgánica de Comunicación a partir de los artículos publicados sobre el tema
}

\author{
Analysis of the impact of the Organic Law of Communication from articles \\ published on the subject
}

\section{Análise do impacto da Lei Orgânica da Comunicação de artigos publicados sobre o assunto}

\author{
Rosa M. Sabando-Delgado ${ }^{\mathrm{I}}$ \\ rosamonserrate@hotmail.com \\ Mario J. Cedeño-Bravo II \\ pegasus_mcb@hotmail.com \\ Yina M. Vélez-Triviño ${ }^{\text {III }}$ \\ yinamaria69@hotmail.com
}

Recibido: 10 de septiembre de $2017 *$ Corregido: 18 de octubre de $2017 *$ Aceptado: 30 noviembre de 2017

I Master en Tecnologías de la Información y de la Comunicación aplicadas a la Educación, Licenciada en Ciencias de la Educación especialidad Castellano y Literatura, Docente de la Universidad San Gregorio de Portoviejo, Portoviejo, Ecuador.

II Especialista en Gerencia Estratégica de la Comunicación Organizacional, Abogado de los Juzgados y Tribunales de la República, Licenciado en Ciencias Sociales y Políticas, Licenciado en Periodismo, Periodista Profesional, Docente de la Universidad San Gregorio de Portoviejo, Portoviejo, Ecuador.

III Diploma Superior en Derecho Procesal Penal, Abogado de los Juzgados y Tribunales de la República, Licenciado en Ciencias Políticas y Sociales, Docente de la Universidad San Gregorio de Portoviejo, Portoviejo, Ecuador. 


\title{
Resumen
}

Desde junio de 2013, en Ecuador, está vigente la Ley Orgánica de Comunicación, una propuesta del gobierno nacional impulsada por el ex-presidente de la República, Rafael Correa Delgado. Esta ley fue aprobada por la Asamblea Nacional con el objetivo de garantizar el acceso y el ejercicio de la comunicación en el país. Existen muchos criterios a favor y en contra de la misma. El presente trabajo tiene como objetivo analizar el impacto de la Ley Orgánica de Comunicación, a partir de los artículos publicados sobre el tema. Para ello se realiza un análisis documental.

Palabras clave: Ley; Ecuador; comunicación.

\begin{abstract}
Since June 2013, in Ecuador, the Organic Communication Law is in force, a proposal of the national government promoted by the former president of the Republic, Rafael Correa Delgado. This law was approved by the National Assembly with the aim of guaranteeing access and exercise of communication in the country. There are many criteria for and against it. The objective of this paper is to analyze the impact of the Organic Law of Communication, based on articles published on the subject. To do this, a documentary analysis is carried out.
\end{abstract}

Keywords: law; Ecuador; communication.

\section{Resumo}

Desde junho de 2013, no Equador, a Lei Orgânica da Comunicação está em vigor, uma proposta do governo nacional promovida pelo ex-presidente da República, Rafael Correa Delgado. Esta lei foi aprovada pela Assembléia Nacional com o objetivo de garantir o acesso e o exercício da comunicação no país. Existem muitos critérios para e contra ele. O objetivo deste trabalho é analisar o impacto da Lei Orgânica da Comunicação, com base em artigos publicados sobre o assunto. Para fazer isso, é realizada uma análise documental.

Palavras chave: lei; Equador; comunicação.

\section{Introducción}

Desde junio de 2013, en Ecuador, está vigente la Ley Orgánica de Comunicación, una propuesta del gobierno nacional impulsada por el ex-presidente de la República, Rafael Correa Delgado. Esta ley 
fue aprobada por la Asamblea Nacional con el objetivo de garantizar el acceso y el ejercicio de la comunicación en el país.

A nivel internacional existen leyes o reglamentos similares: en Argentina se estableció la Ley de Servicios de Comunicación Audiovisual; en Brasil la Ley de Medios de Comunicación democráticos; en Venezuela la Ley de Responsabilidad Social de radio, televisión y medios electrónicos; en Bolivia la Ley General de Telecomunicaciones; y en Colombia por su parte cuenta con el código colombiano de autorregulación. (Sánchez Calderón, y otros, 2016).

La nueva ley de comunicación fue expedida y publicada el 25 de junio de 2013, en el Suplemento del Registro Oficial No. 22, en respuesta de una consulta popular realizada el 7 de mayo de 2011 en la cual el pueblo ecuatoriano decidió que la Asamblea Nacional crease una ley de comunicación que sustente un "Consejo de Regulación donde se normalice la difusión de contenidos de la televisión, radio y publicaciones de prensa escrita los cuales contengan mensajes de violencia, explícitamente sexuales o discriminatorios; y que establezca criterios de responsabilidad ulterior de los comunicadores o medios emisores".

La respuesta constitucional a esta ley es, que ésta debe cumplir con un lineamiento garantista y complementario a lo dispuesto en los art. 18 de la Constitución ecuatoriana, y, en los instrumentos internacionales de protección a la libertad de expresión como es el art 13 de la Convención Americana de Derechos Humanos.

La ley aprobada tiene un cuerpo de ciento diecinueve artículos en seis títulos, veintiséis disposiciones transitorias, seis reformatorias y dos derogatorias. El objeto de la ley es desarrollar, proteger y regular, en el ámbito administrativo, el ejercicio de los derechos a la comunicación establecidos en la constitución. (Asamblea Nacional, s/f).

Reconoce como medios de comunicación social a las empresas y organizaciones públicas, privadas o comunitarias que prestan el 'servicio público de comunicación masiva' usando como herramienta medios impresos o servicios de radio, televisión y audio o vídeo por suscripción, cuyos contenidos puedan ser generados o replicados por internet; sin embargo, aclara que no regula la información u opinión que 'de modo personal' circule a través de la red. 
De igual forma exige a los medios de comunicación, la expedición de códigos deontológicos y el Art. 10 incluye un total de veintinueve normas deontológicas, cuya infracción implica amonestación por parte de la superintendencia de información. Además de una buena cantidad de principios y derechos, resaltan los artículos relacionados con la prohibición de censura previa, responsabilidad ulterior del periodista y del medio, así como el derecho a la información 'verificada, contrastada, precisa y contextualizada. (Asamblea Nacional, s/f).

En el Título III la ley introduce el concepto de 'Sistema de Comunicación social' “Art. 45.Conformación.- El sistema de comunicación Social se conformará por instituciones de carácter público, las políticas y la normativa, así como los actores privados, comunitarios y ciudadanos que se integren voluntariamente a él, de acuerdo al reglamento de esta ley” (Asamblea Nacional,, s/f).

Este sistema tiene como objetivos:

- Articular recursos y capacidades de los actores públicos, comunitarios y privados que conforman el sistema, para lograr el pleno ejercicio de los derechos de la comunicación.

- Desarrollar e implementar mecanismos de planificación pública participativa y descentralizada para la definición, control social así como la adecuación de todas las políticas públicas decomunicación.

- Monitorear y evaluar las políticas públicas y los planes nacionales relativos a los derechos a la comunicación.

- Producir información sobre los avances y dificultades en la aplicabilidad de los derechos de la comunicación.

En este marco y bajo el subtítulo de Institucionalidad para la Regulación y control, la ley crea el Consejo de regulación y Desarrollo de la comunicación, el Consejo consultivo y la Superintendencia de información y comunicación.

Integran el Consejo de regulación un representante de la Función ejecutiva, al igual que uno de los Consejos de igualdad y del Consejo de participación Ciudadana y Control social, de igual modo uno de los Gobiernos Autónomos Descentralizados y uno del Defensor del Pueblo. Su función, junto con el Superintendente de Comunicación e Información consiste principalmente en ejecutar el 
cumplimiento de la ley, para ello tendrá que generar los instrumentos legales que le permitan regular y controlar.

En los temas de desarrollo de política pública, el consejo de regulación podrá contar con una asesoría no vinculante de parte de un Consejo Consultivo, conformado por un representante de los realizadores audiovisuales, otro de los comunicadores sociales, uno por las organizaciones ciudadanas relacionadas a la promoción de la cultura, un representante de los catedráticos de las facultades de comunicación; y uno de los estudiantes de comunicación.

Las siguientes secciones hacen referencia a la regulación y clasificación de contenidos, luego pasa a definir quiénes son medios de comunicación social, entre los cuales incluye a los medios públicos, medios públicos de carácter oficial, medios privados y medios comunitarios. En el caso de los medios públicos incluye una enumeración a sus objetivos. En torno a las obligaciones, entre otras cosas, especifica las restricciones en los que todos los medios se encuentran sujetos en caso de un estado de excepción. Se detallan los espacios obligatorios para el sector público (cadenas nacionales), la distribución equitativa de frecuencias (33\% público, 33\% privado y 34\% comunitario) y en algo explica el progresivo cumplimiento así como la forma de adjudicación. Finaliza con artículos que regulan la difusión de publicidad.

El presente trabajo tiene como objetivo analizar el impacto de la Ley Orgánica de Comunicación a partir de los artículos publicados sobre el tema.

\section{Desarrollo}

En la literatura aparecen disímiles criterios con respecto a la Ley de Comunicación. Por ejemplo la Pontificia Universidad Católica de Ecuador considera que "La nueva ley de comunicación ha puesto en práctica principalmente la potestad de la Superintendencia que en un año y poco más ha emitido resoluciones sancionadoras a medios de comunicación y periodistas en las cuales muy pocas veces se trata de sustentar de forma clara, tomando en cuenta toda la dimensión del derecho a la libertad de expresión, ya que por lo general recae en la subsunción de la regla con el hecho supuesto que se ve a simples rasgos." (Pontificia Universidad Católica del Ecuador, s/f). 
“Al tratarse de la limitación de un derecho humano, es condicional que dicha restricción se establezca por ley, la cual debe ser clara para que no recaiga en una aplicación discrecional así como arbitraria, y pueda distinguirse en cual momento o bajo qué motivo aplicarla. Hay ejemplos en los que no se establecieron las condiciones mínimas de regulación para limitar el derecho a la libertad de expresión conforme al corpus iuris de los instrumentos internacionales formalmente celebrados por el Ecuador. En base a esto también se incluiría que el Estado es totalmente responsable si genera o proporciona medios o recursos para de manera indirecta violentar contra los derechos humanos y en este caso contra la libertad de expresión y la dignidad de los profesionales de la comunicación." (Pontificia Universidad Católica del Ecuador, s/f).

Asimismo Ávila (2013) expone que "Si bien es cierto, en el Ecuador hay una necesidad de organizar un cuerpo legal, en algunos casos insuficiente y caduco, en torno al ejercicio de la comunicación, y esta Ley hace un importante esfuerzo por cumplir con esa necesidad, existen también ciertos reparos, principalmente asociados a la ambigüedad de ciertos conceptos que pueden caer en una suerte de autocensura dentro del ejercicio del periodismo" (Ávila, 2013).

Y considera que la inclusión de principios deontológicos en un ámbito muy general sin especificaciones de procedimientos para sanción, da lugar a interpretaciones diversas por quienes deban ejercer el control y que a simple vista parece una sutil forma de regular la actuación del periodista y del medio de comunicación.

Ávila (2013) hace hincapié en que los objetivos ambiguos han sido criticados por el sector político y los organismos internacionales, entre ellos se encuentran;

- La integración voluntaria ¿qué pasaría con los periodistas que no se integren?

- No especifica qué tipo de instituciones puedan integrar ese sistema

- ¿Los medios privados pueden autoexcluirse?

- Si el sistema no es totalmente incluyente ¿puede tener algún efecto su gestión?

- ¿Cómo se articula para el cumplimiento de las funciones asignadas?

- La distribución de recursos, la planificación de políticas, ¿cuál va a ser su estructura? 
- ¿Cómo se establece un sistema sin la especificación de sus miembros?

"El abordaje teórico hubiese facilitado la discusión del proyecto de ley, ya no solo desde la libertad de expresión, sino de la necesidad de contar con una verdadera representación en la diversidad de intereses al interior del sistema medial, considerado como espacio de deliberación. Lamentablemente el enfoque del proyecto y ahora ley de comunicación del Ecuador considera preferentemente el diagnóstico y sus soluciones inmediatas y omite la proyección de un solvente sistema de comunicación.” (Ávila, 2013).

El derecho a la libertad de expresión no puede ser ampliamente limitado por tanto requiere de una ley clara y taxativa, cuya finalidad de restringir la libertad de expresión sea garantizar la tutela efectiva de quienes han sido lesionados por el abuso de este derecho. Este principio de protección especial recalca la importancia que tiene este derecho para sustentar y sostener los valores democráticos que hacen posible el ejercicio de los demás derechos humanos.

Según la Universidad Pontificia del Ecuador (s/f):

- La nueva ley de comunicación ha generado gran preocupación no solo para los profesionales de la comunicación sino también para los tratadistas y activistas del derecho a la libertad de expresión así como a la sociedad en general, ya que muchos de los principios que contiene este derecho son desarrollados de forma restrictiva y discordante al Sistema Internacional de Protección de los Derechos Humanos, generando así nuevas formas de represión para el ejercicio de este derechos. Es así como el Estado está incumpliendo su obligación de garantía y protección ya que debe adoptar medidas eficaces de protección contra los ataques destinados a callar a quienes ejerzan su derecho a la libertad de expresión. No se puede justificar el silencio a defensores ni comunicadores que están facultados por un sistema democrático.

- La nuevas potestades de regulación y control que emana la ley a dos organismos, deben estar sujetas a los limites sancionatorios y el resultado de sus atribuciones deben ser jurídicamente motivadas basándose en la premisa que "en una sociedad democrática el poder punitivo sólo se ejerce en la medida estrictamente necesaria para proteger los bienes jurídicos fundamentales de los ataques más graves que los dañen o pongan en peligro. Por tanto se reconoce la importancia de ponderar siempre 
la conducta realizada, la forma en la que actuó, el daño causado relacionado a un derecho fundamental y la necesidad de utilizar de manera excepcional medidas sancionatorias.

- La nueva ley de comunicación se ha convertido en una herramienta más que garantista del derecho a la libertad de expresión, una de control, ya que es evidente que la limitación conceptual de este derecho y de sus principios reflejan la conflictividad del Gobierno ecuatoriano y la prensa, que bajo un discurso de intolerancia al abuso de los medios de comunicación se hace necesario restringir y controlar su funcionamiento radicando así restricciones que se configuran en censura previa. Como aporte adicional que enlaza esta conclusión, se ha observado además que los casos revisados se caracterizan por la calificación de las presuntas víctimas, las mismas que representan a dignidades públicas del Estado, y que en los procesos han demostrado su influencia social y mediática.

- Conforme al resultado de las encuestas tomadas de una muestra entre cincuenta periodistas, académicos y profesionales de la comunicación, el 94\% considera que existe una restricción a la libertad de expresión, manifestando que las principales causas para que exista esta limitación son: el no poder expresar las ideas libremente, existe una ley la cual regula los contenidos que los medios de comunicación dan a conocer, no hay acceso adecuado a la información y existe un órgano de regulación y control. Como resultado de esto, manifiestan que hay un orden de importancia de los temas que trata la LOC y entre ellos predomina el establecimiento de sanciones y la regulación del ejercicio de los medios de comunicación, colocando casi al final en orden de prioridades, al tema del cumplimiento de garantías constitucionales referentes al Derecho a la libertad de expresión.

- De los treinta y ocho casos analizados, se encuentran veinte denuncias en la región Costa, siendo estos mayoritarias en la provincia del Guayas y El Oro, mientras que en la Sierra se presentan trece procesos, los de mayor concurrencia en la provincia de Pichincha, y finalmente en la región Amazónica se presentan cinco procesos, divididos entre las provincias de Morona Santiago, Orellana y Sucumbíos.

- De los dieciocho casos revisados, dieciséis de ellos tiene sentencia, de las cuales cinco no han declarado la responsabilidad penal de las personas denunciadas, de igual forma dos por prescripción de la acción, mientras que uno por declaración de nulidad del proceso y dos por no comprobar la existencia del delito; por otro lado existe una sentencia condenatoria por injurias, a la cual el 
sentenciado no logra cumplir su pena de seis meses de prisión por prescripción de la pena. Entre las diez restantes cuatro han sido sentencias condenatorias y de medidas de reparación mientras que las seis restantes han determinado el desistimiento de parte actora.

- Las denuncias presentadas en los dieciocho casos, se debe a la falta cometida de los artículos 489, 491 y 493 del código penal, tipificado como injurias calumniosas, a excepción de los casos: de Morona Santiago que se condena a un representante radiodifusor por el delito de terrorismo y atentar contra el orden público; de Sucumbíos quien inicia un proceso ante el Juez de lo Civil por indemnización por daño moral; y la denuncia presentada por infracciones de los medios de comunicación descrita en el art. 277 de la Ley Orgánica Electoral y de Organizaciones Políticas.

- El análisis para declarar las conductas de los diferentes hechos de cada caso como delito es carente puesto que no existe un detenimiento en las categorías dogmáticas del delito en especial sobre tipicidad y la identificación del bien jurídico vulnerado. De tal manera que los casos en los que se ha declarado la inocencia de los implicados ha sido a razón de las excepciones de forma en el proceso.

- Todos los casos revisados se caracterizan por la calificación de las presuntas víctimas, las mismas que representan a dignidades públicas del Estado, y que en los procesos han demostrado su influencia social y mediática.

Sin embargo, según un estudio realizado en el 2016 por Sánchez Calderón, en el que analizó la opinión de profesionales sobre la producción nacional, publicidad infantil, inversión publicitaria, difusión del tiraje en prensa, entre otros temas que trata la ley, resulta que:

- Los planificadores de medios y creativos publicitarios muestran su aceptación en la mayor parte de los artículos de la Ley Orgánica de Comunicación relacionados con el ámbito publicitario.

- Para los medios impresos y productores audiovisuales, la transparencia de la difusión del tiraje de los periódicos, así como el apoyo a la producción nacional son los artículos más aceptados, ya que facilitan el trabajo y fomentan el desarrollo de la producción nacional.

- Hay artículos que determinan sanciones que finalmente no se cumplen según lo previsto. 
- Es necesario el monitoreo de los canales de televisión permanentemente con el fin de controlar la saturación publicitaria.

- La ley y su reglamento mejoran la actividad publicitaria y fomentan el desarrollo de la actividad en el país.

- Si en algún momento se implementa o cambia algún artículo, se debe analizar la factibilidad física y tecnológica para poder monitorear o controlar su cumplimiento.

Y a criterio de Benavides (2014), la Ley Orgánica de Comunicación, ha creado una nueva forma de llevar la comunicación en Ecuador; se crearon normas que regulan a todos los medios que difunden información como a las personas que van a recibirla; de igual manera se crearon instituciones que sean intermediarios entre la sociedad y los medios, como lo son los defensores de audiencia. Estos son los encargados de recibir quejas, sugerencias, entre otras, así como ayudar a la comunidad para que sus palabras sean escuchadas y sus peticiones aceptadas al igual que respondidas.

"Esta Ley no coarta libertades, únicamente limita la libertad de las personas para que no irrumpa la libertad ajena. Uno no puede hacer uso de un derecho constitucional, violando el mismo derecho que pertenece a otro. Los derechos justamente son para que las personas hagan uso de ellos para su beneficio y el bien común, no se puede permitir que su uso sea para violentar a otros. Por tal razón la nueva ley limita a los medios de comunicación y regula su información, pero no en la manera en la que muchos ciudadanos fueron informados. Se dan parámetros sobre los cuales los medios tienen que basarse, les obliga a respetar la intimidad en ciertos casos, a llevar un mayor control, y da la facilidad a cualquier persona de acceder a estos medios para darse a escuchar. Esta ley obliga a cada medio a realizar un Código Deontológico el cual contiene varios principios que están determinados en el artículo 10 de esta ley. Estos principios se refieren a la dignidad humana, a los grupos de atención prioritaria; y, a lo relacionado con las prácticas de los medios de comunicación social. En la actualidad los medios de comunicación se han tenido que acatar a muchas nuevas reglas que se establecieron. Para mi punto de vista esta Ley fue una creación de gran importancia, ya que la comunicación es un derecho constitucional que afecta y ayuda a todos, por tal motivo es un deber del Estado regular y proteger a los ciudadanos de la información difundida, información que tiene que ser siempre veraz, y la prohibición de que con esto se pueda afectar la dignidad de alguien o se viole su privacidad. Cada 
una de las personas que desea hacer una crítica a esta ley, en primera instancia no debe basarse únicamente en lo que está escrito sino en cómo va a ser usado en la práctica. No se puede dedicar a siempre ver las cosas malas; sino, que aprender a resaltar los lados positivos para que esos sean los que lleven a un progreso en esta nueva área como lo es la comunicación. De igual modo no hacer una crítica por la ideología que se tiene, hay que ser objetivos al momento de analizar normas que van a regir a toda una sociedad. En Sudamérica desde hace unos años se ha tenido más influencia del socialismo, pero esto no puede verse relacionado con estas leyes, ya que con esta ley únicamente lo que se pretendió fue poner orden a todos los medios tanto públicos como privados, para que tomen conciencia que la labor que estos cumplen influye en gran parte al desarrollo del país. Por tal razón en cuanto una norma no nos limite a expresar nuestras ideas sin violar el derecho de otros, según mi pensar creo que es justa y viable para una nueva idea comunicacional ecuatoriana." (Benavides, 2014).

Revelo (2016) puntualiza que esta Ley Orgánica de Comunicación garantiza el acceso a la información de manera activa y responsable, reformula a la comunicación, independientemente de los medios masivos, es una condición básica de las relaciones humanas y socioculturales, es el reconocimiento del "uno y del otro o varios otros", quienes establecen interacciones objetivas y subjetivas que ponen en juego sus valoraciones.

Esta Ley fomenta la participación ciudadana como la promotora en la construcción de un nuevo lenguaje comunicacional informativo-periodístico, basada en el compromiso y solidaridad estableciendo verdaderos cauces de participación a lo largo de todo del proceso comunicativo. Los productores audiovisuales están fortalecidos con esta ley, tienen la posibilidad de crear y recrear formas y estilos para involucrar activamente al espectador para sus fines, esta ley pretende que los medios de comunicación social dejen de ser "utilitarios" o "instrumentos de manipulación” de grandes grupos de poder que "aboban e idiotizan", que imponen a través de sus mensajes nuevos comportamientos y estilos de vida a la población; que los grandes medios dejen de mirar "peyorativamente a las masas". Se sabe que estos medios son industrias culturales que construyen una mirada del mundo desde sus escenarios y sus propias producciones, recrean actores y contenidos emocionalmente y en serie. Estos medios ofrecen espacios públicos desde lo urbano y cotidiano que son propios de sociedades modernas capitalistas que incitan al consumo.” (Revelo, 2016). 


\section{Conclusiones}

Mediante la literatura se puede comprobar que existen disímiles criterios sobre la ley de Comunicación en el Ecuador. Muchos reconocen que regula el accionar informativo y comunicacional permitiendo fomentar de manera democrática, activa y participativa el ejercicio de la opinión pública y otros cuestionan la libertad de expresión, entre otros aspectos.

\section{Referencias Bibliográficas}

Asamblea Nacional, s/f. Constitución del Ecuador. [En línea] s/f. [Citado el: 05 de julio de 2017.] http://www.asambleanacional.gov.ec/documentos/constitucion_de_bolsillo.pdf

Ávila, C. 2013. El sistema de comunicación en la Ley Orgánica de Comunicación del Ecuador: Un análisis desde el enfoque de las teorías de sociedad y masas de McQuail. Chasqui No. 124. 2013

Benavides, A.B. 2014. Análisis a la ley de comunicación, s. 1.: trabajo para la obtención del título de abogada, 2014

Pontificia Universidad Católica del Ecuador, s/f. La nueva ley de comunicación y su aplicación para el ejercicio periodístico y el derecho a la libertad de expresión en el Ecuador. [En línea] s/f. [Citado $\begin{array}{lllll}\text { el: } 03 & \text { julio diciembre }\end{array}$ http://www.uasb.edu.ec/documents/62017/1434654/La+nueva+Ley+de+Comunicaci\%C3\%B3n+y+ su+aplicaci\%C3\%B3n+para+el+ejercicio+period\%C3\%ADstico.pdf/d68bf7ed-d741-4137-8fb9$1 \mathrm{cf} 850896 \mathrm{~d} 76$

Revelo, J. 2016. Num.1-2015-Art.9 I. Miradas a los más media y a la Ley de Comunicación del Ecuador. Ecos de la Academia. 2016

Sánchez Calderón, H, Yánez Balarezo, S y Martínez Segovia, M. 2016. Impacto de la Ley Orgánica de Comunicación en la Publicidad Ecuatoriana. Prisma Social, núm. 17. 2016 